\title{
Application of Out-of-Hospital Extended Nursing in Brace Treatment of Patients with Idiopathic Scoliosis
}

\author{
Feng-Xiang ZHU ${ }^{1}$, Hai-Yan SHAO ${ }^{2}$, Hong-Ying ZHU ${ }^{1}$, Xiao-Ling HUANG ${ }^{1}$, Ling TANG ${ }^{3}$ \\ ${ }^{1}$ Department of Spine and Spinal Cord Surgery, Shandong Wendeng Osteopathic Hospital, Weihai, Shandong, 264400, \\ China; ${ }^{2}$ Nursing Department, Shandong Wendeng Orthopaedic Hospital, Weihai, Shandong, 264400, China; ${ }^{3}$ Nursing \\ Department, the Third Affiliated Hospital of Beijing University of Chinese Medicine, Beijing, 100029, China
}

\begin{abstract}
Objective: To investigate the effect of out-of-hospital extended nursing on the compliance behaviors and therapeutic effect of brace treatment of patients with idiopathic scoliosis.

Methods: 54 patients with idiopathic scoliosis between February 2015 and December 2017 were randomly divided into control group and observation group. Patients in the control group received pelvic suspension traction, gymnastic exercises, and brace wear at discharge, on the basis of which patients in the observation group were added with extended care outside the hospital. The compliance behaviors and the changes of scoliosis angle (Cobb angle) of patients in the 2 groups were evaluated.

Results: Compared with the control group, patients in the observation group had better compliance behaviors in completion status of functional exercise $\left(\chi^{2}=5.594, P=0.018\right)$, brace wear $\left(\chi^{2}=6.171, P=0.013\right)$, subsequent visit on time $\left(\chi^{2}=9.247, P=0.002\right)$. Cobb angle was improved significantly in both groups at the last follow-up compared with that on admission, and the improvement was more significantly in the observation group $(P<0.001)$.

Conclusion: Through the implementation of out-of-hospital extended nursing, the compliance behaviors and clinical effect of brace treatment for idiopathic scoliosis patients are obviously improved, and this active nursing model is worth popularizing in clinic.
\end{abstract}

Keywords: Out-of-hospital extended care; Idiopathic scoliosis; Brace treatment; Compliance behaviors

\section{Introduction}

Idiopathic scoliosis is a common disease of the spine where one or more segments deviate from the middle of the body on the coronal surface ${ }^{[1]}$, characterized by a curvature of the spinal deformity, most commonly occurred in children and adolescents ${ }^{[2]}$. It will not only cause body deformity, back pain, mobility inconvenience,

Ling TANG, female, director of nursing department of the Third Affiliated Hospital of Beijing University of Chinese Medicine, Beijing I00029, China

E-mail: zydsyhlb@126.com

Received:April 9 2019, Revised: June 3 2019,Accepted: June 25 2019, Online:

August 152019 psychological burden and so on, more importantly, affect the heart and lung functions of patients ${ }^{[3-4]}$. Therefore, early conservative treatment is of great significance to the disease $^{[5-10]}$. However, the poor compliance behaviors of teenagers after discharge seriously affects the therapeutic effect $^{[11]}$, in order to study the rehabilitation training of patients after discharge and the compliance with the correct brace wear, and to further improve the compliance behaviors of patients after discharge, we implemented outof-hospital extended nursing for patients with idiopathic 
scoliosis between February, 2015 and December 2017, and the clinical effect was satisfactory, which was reported below.

\section{Materials and Methods}

\section{General Information}

A total of 54 children who suffered idiopathic scoliosis admitted to our department from February, 2015 to December 2017 were enrolled. After admission, the $\mathrm{X}$-Ray was perfected to make a definite diagnosis, and the patients were divided into observation group (27 cases) and control group (27 cases) according to the random double-blind method of admission order. In the observation group, there were 8 males and 19 females, aged 8-17 years old with an average age of 9.7. There were 2 cases of thoracic scoliosis, 4 cases of lumbar scoliosis, 21 case of thoracolumbar scoliosis. In the control group, there were 9 males and 18 females, aged 8-17 years old with an average age of 9.1. There were 2 cases of thoracic scoliosis, 5 cases of lumbar scoliosis, and 20 cases of thoracolumbar scoliosis. All included patients were followed up for 12-50 months, with an average of 26 months. There was no statistical difference in the above general data between the two groups $(P>0.05)$.

\section{Inclusion criteria}

(1) Patients meet the diagnostic criteria for adolescent idiopathic scoliosis ${ }^{[12]}$. (2) After admission, all patients underwent anteroposterior X-ray examination in standing position, and the Cobb Angle (the intersection angle between the vertical line connecting the upper and lower edges of the vertebral pedicle) exceeded $10^{\circ}$. (3) Patients meet the clinical diagnostic criteria of idiopathic scoliosis.

\section{Treatment methods}

After admission, all patients were given pelvic suspension traction, 30-60 minutes each time, 6-8 times a day. Orthopaedic gymnastic exercise (Yanfei-type lumbar dorsal muscle function exercise) was needed to be cooperated $w_{i t h}{ }^{[13]}$. The specific movements were as follows: The patient was in prone position, kept the lower limbs in the straight position, and placed two hands at the sides of the body. When the patient raised his/her head, his/her upper body stretched backwards, and the lower extremities stretched straightly and backwards, and the waist stretched as far as possible like a flying swallow, 1020 times for a set of movement, 5-10 sets daily. Patients wore the brace after discharge. The brace was made of polyethylene with good biocompatibility and adjustable temperature by guidance of clinical physician and professional technicians. Within 1-2 weeks after discharge, patients were asked to wear it for about 6 hours daily, and gradually increase the wear time after adapting to it, and to more than 16 hours at about 1 month after discharge. Besides, patients were required to recheck X-ray regularly.

\section{Extended nursing}

Department organized out-of-hospital extended care team consisting of specialized nursing staff and competent doctors. During hospitalization, patients received routine care of spinal surgery. After discharge from hospital, patients in the control group were given telephone return visit once only at 1 month after discharge. While patients in the observation group were carried out with extended care. Guidance should be given to patients in terms of functional exercise, brace wear and subsequent visit time. Patients are followed up weekly by telephone within 3 months after discharge, outpatient visit once every 2 weeks, followed by timely telephone follow-up according to the patient's situation.

\section{Evaluation indexes}

The compliance behaviors (completion status of functional exercise, brace wear, on-time subsequent visit) and scoliosis angle (Cobb angle) of patients of 2 groups were evaluated after the implementation of out-of-hospital extended care for 12 months.

\section{Statistical analysis}

SPSS 17.0 statistic software was used for statistical analysis. The measurement data (Cobb angle) were expressed as mean \pm standard deviation $(\bar{x} \pm s)$, and were analyzed using $t$ test. The enumeration data (completion of functional exercise, brace wear, subsequent visit on time) was expressed as percentage (\%), and the rate between groups was analyzed by $\chi^{2}$ test. $P<0.05$ was considered to be statistically significant. 
Table 1 Comparison of compliance behaviors between the 2 groups [ $n(\%)]$

\begin{tabular}{lcccc}
\hline Groups & Number & Functional exercise & Brace wear & Subsequent visit on time \\
\hline Observation group & 27 & $25(92.6)$ & $24(88.9)$ & $26(96.3)$ \\
Control group & 27 & $18(66.7)$ & $16(59.3)$ & $17(63.0)$ \\
$\chi^{2}$ & & 5.594 & 6.171 & 9.247 \\
$P$ & & 0.018 & 0.013 & 0.002 \\
\hline
\end{tabular}

Table 2 Cobb angle changes of 2 groups $(\bar{x} \pm s)$

\begin{tabular}{lcccc}
\hline Groups & Number & On admission $\left(^{\circ}\right)$ & At discharge $\left(^{\circ}\right)$ & Last follow-up $\left(^{\circ}\right)$ \\
\hline Observation group & 27 & $34.8 \pm 4.1$ & $15.3 \pm 3.9$ & $9.4 \pm 2.7$ \\
Control group & 27 & $33.1 \pm 4.0$ & $16.8 \pm 3.7$ & $13.9 \pm 3.1$ \\
$t$ & & 1.542 & 1.450 & 5.688 \\
$P$ & & $>0.05$ & $>0.05$ & $<0.001$ \\
\hline
\end{tabular}

\section{Results}

\section{Comparison of compliance behaviors of the 2 groups}

Compared with the control group, patients in the observation group had better compliance behaviors in completion status of functional exercise $\left(\chi^{2}=5.594, P=\right.$ $0.018)$, brace wear $\left(\chi^{2}=6.171, P=0.013\right)$, subsequent visit on time $\left(\chi^{2}=9.247, P=0.002\right)$, as shown in Table 1 .

\section{Scoliosis angles (Cobb angle) changes of 2 groups}

There was no difference between two groups before admission and at discharge with regard to Cobb angle $(P>$ 0.05). However, Cobb angle was smaller significantly in both groups at the last follow-up compared with that on admission, and the improvement was more significantly in the observation group $(P<0.001)$. (Table 2$)$

\section{Discussion}

From table 1, it can be seen that completion status of functional exercise, brace wear and on-time return visit is significantly better in the observation group than the control group, indicating that out-of-hospital extended nursing can obviously improve the compliance behaviors of discharged patients. Idiopathic scoliosis is commonly occurring in children and adolescents, and patients can't be hospitalized for long periods of time because of their studies or other reasons, while the rehabilitation of idiopathic scoliosis is a long-term process that requires perseverance ${ }^{[14]}$. Therefore, out-of-hospital extended care is doubly important when patients may feel uncomfortable and want to give up at the beginning of wearing brace ${ }^{[15-16]}$, or when they feel bored and can't persist for a long time during the period of rehabilitation training, or when medical staff can not pay timely attention to patients' psychological dynamics and rehabilitation training situation. Out-of-hospital extended care can give full play to the therapeutic effect, including increasing patient compliance and improving the degree of idiopathic scoliosis. This, in turn, increases patients' confidence of treatment, and make patients with a positive attitude receive and cooperate with the treatment, thus obtaining more therapeutic benefits.

For most patients with idiopathic scoliosis which are currently clinical common disease, conservative traction treatment is used in most cases, which is one of the important methods to treat idiopathic scoliosis in clinic at present, and can reduce the pain of operation for patients. At the same time, good out-of-hospital extended care can further improve patient compliance ${ }^{[17-18]}$, make patients insist on doing rehabilitation training and correct brace wear, eventually significantly improving the degree of scoliosis (Cobb angle) and the quality of life of the patients ${ }^{[19]}$. From table 2, it can be seen that Cobb angle is smaller at the last follow-up of the 2 groups (1 year after discharge) 
than when discharged from hospital, the improvement was more obvious in the observation group compared with the treatment group $(P<0.05)$, indicating that out-of-hospital extended nursing was conducive to correct scoliosis.

In this study, the data of the patients with idiopathic scoliosis treated in our hospital were analyzed, and the outstanding advantages of extended nursing in helping patients in rehabilitation could be found, and extended nursing could meet the needs of patients' nursing after discharge, and make up for the shortage of health education when discharged from hospital. Moreover, it obviously improves the compliance behaviors of patients' rehabilitation training, and this active nursing model strengthens the work enthusiasm of nursing staff, which is thus worth popularizing in clinic.

\section{References}

1 Wang Y, Zhu ZZ, Qiu Y, et al. Effection of thoracic curve on cardiac structure and function in patients with adolescent idiopathic scoliosis. Chin J Spine Spinal Cord, 2016, 26(8): 723-728.

2 Yaman O, Dalbayrak S. Idiopathic scoliosis. Turk Neurosurg. 2014, 24(5): 646-657.

3 Rüwald JM, Eymael RL, Upenieks J, et al. An Overview of the Current State of Pediatric Scoliosis Management. Z Orthop Unfall, 2019. [Epub ahead of print]

4 Zhang BB. Relationship between adolescent idiopathic scoliosis and pelvis. Chin J Orthop Surg, 2016, 24(13): 1198-1201.

$5 \mathrm{Wu}$ P, Li M. Clinical treatment of adolescent idiopathic scoliosis. Mod Med Health, 2015, 31(17): 2618-2620.

6 Yang J, Skaggs DL, Chan P, et al. High Satisfaction in Adolescent Idiopathic Scoliosis Patients on Enhanced Discharge Pathway. J Pediatr Orthop, 2019. [Epub ahead of print]

7 Du JY, Jiang B, Zhao ZJ. Bracing treatment combined with postural training in adolescent idiopathic scoliosis. J Clin Orthop, 2018, 21(6): 647-650.

8 Zeng XD. Clinical value of brace combined with orthopaedic gymnastic exercise in the treatment of adolescent idiopathic scoliosis. Contemp Med, 2016, 22(31): 35-36.

9 Zhang J, Fan YC, Wang ZG, et al. Effect of domestic medical spinal correction bed on pa-tients with AIS. Chin J Disabil Med, 2016, 24(2): 2-4.

$10 \mathrm{Ma}$ YL. Discussion on the application effect of orthotics in the treatment of adolescent idiopathic scoliosis. Front Med, 2017, 7(29): 66-67.

11 Karol LA, Virostek D, Felton K, et al. Effect of Compliance Counseling on Brace Use and Success in Patients with Adolescent Idiopathic Scoliosis. J Bone Joint Surg Am, 2016, 98(1): 9-14.

12 Wang S. Clinical observation of bone-setting massage combined with traction in the treatment of adolescent idiopathic scoliosis. Chin Naturopathy, 2019, 27(11): 26-28.

13 Yang YY. Application and effect analysis of path functional exercise in orthopaedic rehabilitation nursing. China Disabil Med, 2016,24 (11): 112-114.

14 Pellios S, Kenanidis E, Potoupnis M, et al. Curve progression 25 years after bracing for adolescent idiopathic scoliosis: long term comparative results between two matched groups of 18 versus 23 hours daily bracing. Scoliosis Spinal Disord, 2016, 11: 3 .

15 Lou XH, Li P, Liu Y. Nursing of the improved cheneau brace to treat 120 adolescent idipathic scoliosis patients. Chin J Nurs, 2006, 41(2): 116-117.

16 Guo LM, Shu J, Lao HC, et al. Analysis on the treatment of idiopathic scoliosis in adolescents. Chin J Orthop Surg, 2015, 23(11): 967-971.

17 Aulisa AG, Guzzanti V, Marzetti E, et al. Brace treatment in juvenile idiopathic scoliosis: a prospective study in accordance with the SRS criteria for bracing studies - SOSORT award 2013 winner. Scoliosis, 2014, 9: 3.

18 Chalmers E, Lou E, Hill D, et al. An advanced compliance monitor for patients undergoing brace treatment for idiopathic scoliosis. Med Eng Phys, 2015, 37(2): 203-209.

19 Bidari S, Kamyab M, Ahmadi A, et al. Effect of exercise on static balance and Cobb angle during the weaning phase of brace management in idiopathic scoliosis and hyperkyphosis: A preliminary study. J Back Musculoskelet Rehabil, 2019, 32(4): 639-646. 